\title{
Character Sums Attached to Finite Reductive Groups
}

\author{
Naomichi SAITO and Ken-ichi SHINODA
}

Sophia University

\section{Introduction.}

In [12], Kondo determined the value of Gaussian sum for every irreducible representation of $G L_{n}(q)$ and Macdonald also treated this problem in [14]. Recently in a series of papers, Kim-Lee [4], Kim ([5], [6], [7], [8], [9], [10], [11]), and Lee-Park [13] determined the values of Gaussian sums for one-dimensional representations of finite classical groups and $G_{2}(q)$.

In this note, we firstly show that a character sum over a finite reductive group associated with the generalized character $R_{\mathbf{T}, \theta}$ of Deligne-Lusztig is reduced to a character sum over a torus. Applying this result to Gaussian sums and Kloosterman sums attached to finite classical groups, we obtain explicit formulae of these sums related with $R_{\mathbf{T}, \theta}$, when $\pm R_{\mathbf{T}, \theta}$ is irreducible. Also combining this result with the Davenport-Hasse type relations of Kloosterman sums and unitary Kloosterman sums proved in [2], we can explicitly determine the values of these sums for every irreducible character if the rank of the group is low. As an example, we give a table of Gaussian sums attached to $S p_{4}(q)$, with $q$ odd. In Section 3, Kloosterman sums over $G L_{n}(q)$ are considered, and the properties and conjectures of these sums for unipotent characters are given.

The second named author would like to express his sincere gratitude to Masao Koike and Charles W. Curtis for their valuable discussions and comments.

NotAtion. We shall use the similar notation as in [2]. In particular $\mathbb{F}_{q}$ denotes a finite field with $q$ elements, and $\mathbb{F}_{q^{m}}$ the extension field of degree $m$ of $\mathbb{F}_{q}$, contained in a fixed algebraic closure $\overline{\mathbb{F}}_{q}$ of $\mathbb{F}_{q} . C_{m}=\left\{\alpha \in \mathbb{F}_{q^{2 m}}: \alpha^{q^{m}+1}=1\right\}$ is the cyclic group of order

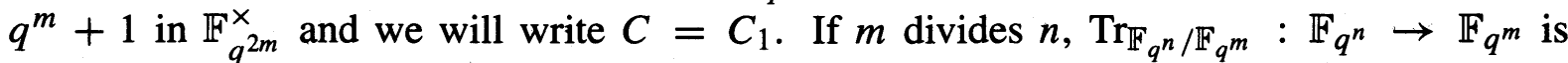
the trace map. We fix a nontrivial additive character $\chi$ of $\mathbb{F}_{q}$ throughout this paper, and put $\chi^{(m)}=\chi \circ \operatorname{Tr}_{\mathbb{F}_{q^{m} / \mathbb{F}_{q}}}$, the canonical lift of $\chi$ to $\mathbb{F}_{q^{m}}$. For a multiplicative character $\pi$ of $\mathbb{F}_{q}^{\times}$, the sum

$$
K(\chi, \pi, a)=\sum_{s t=a} \chi(s+t) \pi(s), \quad a \in \mathbb{F}_{q}^{\times}
$$

Received February 4, 1999 
is called a Kloosterman sum and we denote $K(\chi, \pi)=K(\chi, \pi, 1)$. Also for a character $\varphi$ of $C$, the sum

$$
J(\chi, \varphi)=\sum_{\alpha \in C} \chi\left(\alpha+\alpha^{-1}\right) \varphi(\alpha)
$$

is called a unitary Kloosterman sum (cf. [2]). These sums are defined over any finite fields, and for $\mathbb{F}_{q^{m}}$, we denote $K_{m}\left(\pi_{m}, a\right)=K\left(\chi^{(m)}, \pi_{m}, a\right), K_{m}\left(\pi_{m}\right)=K\left(\chi^{(m)}, \pi_{m}\right)$ and $J_{m}\left(\varphi_{m}\right)=$ $J\left(\chi^{(m)}, \varphi_{m}\right)$, where $\pi_{m}$ (resp. $\left.\varphi_{m}\right)$ is a character $\mathbb{F}_{q^{m}}^{\times}\left(\right.$resp. $\left.C_{m}\right)$ and $a \in \mathbb{F}_{q}^{\times}$.

\section{Character sum with the generalized character of Deligne-Lusztig.}

1.1. Let $\mathbf{G}$ be a connected reductive algebraic group defined over $\mathbb{F}_{q}$, with Frobenius map $\sigma$, and let $G=\mathbf{G}^{\sigma}$ be the finite group consisting with elements in $\mathbf{G}$ fixed by $\sigma$. Let $R$ be an ordinary representation of $G, R: G \rightarrow G L_{m}(\mathbb{C})$, and $\psi$ a complex-valued class function on $G$, which depends only on the semisimple part of each element in $G$. We consider the sum

$$
W_{G}(R, \psi)=\sum_{g \in G} R(g) \psi(g) .
$$

If there is no afraid of confusion, we shall simply write $W(R)$ for $W_{G}(R, \psi)$. If $R$ is irreducible, then $W(R)=w(R) I_{m}$ for some complex number $w(R)$, where $I_{m}$ is the identity matrix of degree $m=\operatorname{deg} R$. Let $\tau_{W}\left(\chi_{R}\right)$ be the trace of $W(R)$, where $\chi_{R}$ is the character of $R$. Then we can extend $\tau_{W}$ to a complex valued function on the Grothendieck group of generalized characters of $G$ and we have $\tau_{W}\left(\chi_{R}\right)=w(R) \operatorname{deg} R$, if $R$ is irreducible. Hereafter we write $w\left(\chi_{R}\right)$ instead of $w(R)$.

1.2. Let $\mathbf{T}$ be a $\sigma$-stable maximal torus of $\mathbf{G}, \theta$ a character of $T=\mathbf{T}^{\sigma}$ and $R_{\mathbf{T}, \theta}$ the generalized character of Deligne-Lusztig corresponding to $\mathbf{T}$ and $\theta$. We recall the following properties of the generalized character $\boldsymbol{R}_{\mathbf{T}, \theta}$ (cf. [3] or [1]):

- (Character formula) Let $g \in G$ have Jordan decomposition $g=s u=u s$, where $s$ is semisimple and $u$ is unipotent. Then

$$
R_{\mathbf{T}, \theta}(g)=\frac{1}{\left|\mathbf{C}^{0}(s)^{\sigma}\right|} \sum_{\substack{x \in G \\ x^{-1} s x \in T}} \theta\left(x^{-1} s x\right) Q_{x \mathbf{T} x^{-1}}^{\mathbf{C}^{0}(u)}(u),
$$

where $Q_{x \mathbf{T} x^{-1}}^{\mathbf{C}^{0}(s)}$ is the Green function of the connected component of the centralizer of $s$ in $\mathbf{G}$, denoted by $\mathbf{C}^{0}(s)$, corresponding to $x \mathbf{T} x^{-1}$.

$$
\sum_{\substack{u \in G \\ \text { unipotent }}} Q_{\mathbf{T}}^{\mathbf{G}}(u)=|G: T|
$$

- If the character $\theta$ of $T$ is in general position, then $\varepsilon_{\mathbf{G}} \varepsilon_{\mathbf{T}} R_{\mathbf{T}, \theta}$ is an irreducible character of $G$ of degree $|G: T|_{p^{\prime}}$, where $|G: T|_{p^{\prime}}$ denotes the $p^{\prime}$-part of $|G: T|$ and $\varepsilon_{\mathbf{G}}=$ $(-1)^{\text {rel.rank } G}$ and similarly $\varepsilon_{\mathbf{T}}=(-1)^{\text {rel.rank } \mathbf{T}}$.

Now we can prove 
THEOREM. With the notation as above, we have

$$
\tau_{W}\left(R_{\mathbf{T}, \theta}\right)=\frac{|G|}{|T|} \sum_{t \in T} \theta(t) \psi(t) .
$$

In particular, if $\theta$ is in general position, then

$$
w\left(\varepsilon_{\mathbf{G}} \varepsilon_{\mathbf{T}} R_{\mathbf{T}, \theta}\right)=\varepsilon_{\mathbf{G}} \varepsilon_{\mathbf{T}}|G|_{p} \sum_{t \in T} \theta(t) \psi(t)
$$

Proof. One has

$$
\begin{aligned}
\tau_{W}\left(R_{\mathbf{T}, \theta}\right) & =\sum_{g \in G} R_{\mathbf{T}, \theta}(g) \psi(g) \\
& =\sum_{s \in G, \text { s.s. }}\left\{\sum_{u \in \mathbf{C}(s)^{\sigma}, \text { unip. }} R_{\mathbf{T}, \theta}(s u)\right\} \psi(s),
\end{aligned}
$$

where $\mathbf{C}(s)$ is the centralizer of $s$ in $\mathbf{G}$. Now using the first and the second properties of $R_{\mathbf{T}, \theta}$ cited above, the sum inside the parenthesis becomes

$$
\begin{aligned}
\sum_{u \in \mathbf{C}(s)^{\sigma}, \text { unip. }} \frac{1}{\left|\mathbf{C}^{0}(s)^{\sigma}\right|} \sum_{\substack{x \in G \\
x^{-1} s x \in T}} Q_{x \mathbf{T} x^{-1}}^{\mathbf{C}^{0}(s)}(u) \theta\left(x^{-1} s x\right) \\
=\frac{1}{\left|\mathbf{C}^{0}(s)^{\sigma}\right|} \sum_{\substack{x \in G \\
x^{-1} s x \in T}}\left\{\sum_{u \in \mathbf{C}(s)^{\sigma}, \text { unip. }} Q_{x \mathbf{T} x^{-1}}^{\mathbf{C}^{0}(s)}\right\} \theta\left(x^{-1} s x\right) \\
=\sum_{\substack{x \in G \\
x^{-1} s x \in T}} \frac{1}{\left|x T x^{-1}\right|} \theta\left(x^{-1} s x\right) .
\end{aligned}
$$

Here we used the fact that every unipotent element in $\mathbf{C}(s)$ lies in $\mathbf{C}^{0}(s)$, due to Springer and Steinberg. Now changing the variable from $s$ to $t$ by putting $x^{-1} s x=t$, we obtain the required result, since $s$ is uniquely determined by $t$ and $x$. The formula for $w\left(\varepsilon_{\mathbf{G}} \varepsilon_{\mathbf{T}} R_{\mathbf{T}, \theta}\right)$ follows from the third property of $R_{\mathbf{T}, \theta}$.

\section{Gaussian sums attached to finite reductive groups.}

2.1. We use the same notation as in the previous section. Let $\rho$ be a modular representation of $G, \rho: G \rightarrow G L_{n}\left(\mathbb{F}_{q^{m}}\right)$, where $\mathbb{F}_{q^{m}}$ is a finite extension field of $\mathbb{F}_{q}$ contained in $\overline{\mathbb{F}}_{q}$. We define the class function $\psi$ by

$$
\psi(g)=\chi\left(\operatorname{Tr}_{\mathbb{F}_{q^{m}} / \mathbb{F}_{q}}(\operatorname{tr} \rho(g))\right),
$$

where $\operatorname{tr} \rho(g)$ is the trace of the matrix $\rho(g)$. Then $W_{G}(R, \psi)$ is called the Gaussian sum attached to $G, R$ and $\rho$.

2.2. Unitary groups. Let $\mathbf{G}=G L_{n}\left(\overline{\mathbb{F}}_{q}\right)$ and let $\sigma: \mathbf{G} \rightarrow \mathbf{G}$ be the Frobenius map defined by $\sigma(g)={ }^{t} F(g)^{-1}$, where $F(g)=\left(g_{i j}^{q}\right)$ for $g=\left(g_{i j}\right)$ and ${ }^{t} F(g)$ is the transpose 
of $F(g)$. Then $G=\mathbf{G}^{\sigma}$ is called a unitary group, denoted by $U_{n}(q)$, and is a subgroup of $G L_{n}\left(q^{2}\right)$, since $\sigma^{2}=F^{2}$. Let $\mathbf{T}$ be the diagonal matrices of $\mathbf{G}$ and $W=N_{\mathbf{G}}(\mathbf{T}) / \mathbf{T}$ be the Weyl group. $W$ is isomorphic to the symmetric group $S_{n}$ on $n$ letters and by the definition of $\sigma, \sigma$ acts trivially on $W$.

The $G$-conjugacy classes of $\sigma$-stable maximal tori are parametrized by the ( $\sigma$-twisted) conjugacy classes of $W$. If $\mathbf{T}_{w}$ is a $\sigma$-stable maximal torus corresponding to $w \in W$ we may assume that $T_{w}=\mathbf{T}_{w}^{\sigma}$ is isomorphic to $\left\{t \in \mathbf{T}: \dot{w} t \dot{w}^{-1}=\sigma(t)\right\}$, where $\dot{w}$ is a representative of $w$ in $N_{G}(\mathrm{~T})$. On the other hand the conjugacy classes of $S_{n}$ are determined by the cycle types and correspond bijectively to the partitions of $n$, the set of which is denoted by $\mathcal{P}_{\boldsymbol{n}}$. Let $\mathbf{T}_{\lambda}$ be a maximal torus corresponding to a partition $\lambda \in \mathcal{P}_{\boldsymbol{n}}$. Let $\lambda_{01}, \lambda_{02}, \cdots, \lambda_{0 r_{0}}$ be the even parts of $\lambda$ and let $\lambda_{11}, \lambda_{12}, \cdots, \lambda_{1 r_{1}}$ be the odds parts, so that $r_{0}+r_{1}$ is the length of $\lambda$ and $r_{0}$ is the relative rank of $T_{\lambda}$. For $\lambda_{0 i},\left(1 \leq i \leq r_{0}\right)$, let $t_{0 i}(\alpha)=$ $\operatorname{diag}\left(\alpha, \alpha^{-q}, \cdots, \alpha^{(-q)^{\lambda_{0 i}-1}}\right)$, where $\alpha \in \mathbb{F}_{q^{\lambda_{0 i}}}^{\times}$and also for $\lambda_{1 j},\left(1 \leq j \leq r_{1}\right)$, let $t_{1 j}(\beta)=$ $\operatorname{diag}\left(\beta, \beta^{-q}, \cdots, \beta^{(-q)^{\lambda_{1 j}-1}}\right)$, where $\beta \in C_{\lambda_{1 j}}$. Moreover put $t\left(\alpha_{1}, \cdots, \alpha_{r_{0}} ; \beta_{1}, \cdots, \beta_{r_{1}}\right)=$ $\operatorname{diag}\left(t_{01}\left(\alpha_{1}\right), \cdots, t_{0 r_{0}}\left(\alpha_{r_{0}}\right), t_{11}\left(\beta_{1}\right), \cdots, t_{1 r_{1}}\left(\beta_{r_{1}}\right)\right)$. Then $T_{\lambda}=\mathbf{T}_{\lambda}^{\sigma}$ is isomorphic to, and we identify with, the diagonal subgroup

$$
\left\{t\left(\alpha_{1}, \cdots, \alpha_{r_{0}} ; \beta_{1}, \cdots, \beta_{r_{1}}\right): \alpha_{i} \in \mathbf{F}_{q^{\lambda_{0 i}}}^{\times}, \beta_{j} \in C_{\lambda_{1 j}}\right\}
$$

and hence

$$
T_{\lambda} \simeq \prod_{i=1}^{r_{0}} \mathbb{F}_{q^{\lambda_{0 i}}}^{\times} \times \prod_{j=1}^{r_{1}} C_{\lambda_{1 j}}
$$

Let $\pi_{i}$ and $\varphi_{j}$ be characters of $\mathbb{F}_{q^{\lambda_{0 i}}}^{\times}$and $C_{\lambda_{1 j}}$, respectively, and $\theta$ be the character of $T_{\lambda}$ defined by $\theta\left(t\left(\alpha_{1}, \cdots, \alpha_{r_{0}} ; \beta_{1}, \cdots, \beta_{r_{1}}\right)\right)=\prod_{i} \pi_{i}\left(\alpha_{i}\right) \prod_{j} \varphi_{j}\left(\beta_{j}\right)$.

Finally let $\chi^{\prime}=\chi \circ \operatorname{Tr}_{\mathbb{F}_{q^{2}} / \mathbb{F}_{q}}$ and $\rho$ be the inclusion of $G$ into $G L_{n}\left(q^{2}\right)$. Then we can show that $\chi^{\prime}\left(\operatorname{tr} \rho\left(t\left(\alpha_{1}, \cdots, \alpha_{r_{0}} ; \beta_{1}, \cdots, \beta_{r_{1}}\right)\right)\right)=\prod_{i} \chi^{\left(\lambda_{0 i}\right)}\left(\alpha_{i}+\alpha_{i}^{-1}\right) \prod_{j} \chi^{\left(\lambda_{1 j}\right)}\left(\beta_{j}+\beta_{j}^{-1}\right)$. Combining these results with (1.2), we have

Proposition. With the notation as above

$$
\tau_{W}\left(R_{\mathbf{T}_{\lambda}, \theta}\right)=\left|G: T_{\lambda}\right| \prod_{i=1}^{r_{0}} K_{\lambda_{0 i}}\left(\pi_{i}\right) \times \prod_{j=1}^{r_{1}} J_{\lambda_{1 j}}\left(\varphi_{j}\right) .
$$

In particular, if $\theta$ is in general position,

$$
w\left((-1)^{n+r_{0}} R_{\mathbf{T}_{\lambda}, \theta}\right)=(-1)^{n+r_{0}} q^{\left(\begin{array}{c}
n \\
2
\end{array}\right)} \prod_{i=1}^{r_{0}} K_{\lambda_{0 i}}\left(\pi_{i}\right) \times \prod_{j=1}^{r_{1}} J_{\lambda_{1 j}}\left(\varphi_{j}\right) .
$$

EXAMPLE. $U_{2}(q)$ Let $\varphi$ be a character of $C$ and $\varphi^{(2)}$ be the canonical lift of $\varphi$ to $\mathbb{F}_{q^{2}}^{\times}$. Let $J(\varphi)=J(\chi, \varphi)$ and $K_{2}\left(\varphi^{(2)}\right)=K\left(\chi^{(2)}, \varphi^{(2)}\right)$. Then by Theorem 2 in [2], we have

$$
K_{2}\left(\varphi^{(2)}\right)=-J(\varphi)^{2}+2 q \varphi(-1)
$$


Under the same notation as above, we have

$$
\begin{aligned}
\tau_{W}\left(R_{\mathbf{T}_{(1,1),(\varphi, \varphi)}}\right) & =q(q-1) J(\varphi)^{2} \\
\tau_{W}\left(R_{\mathbf{T}_{(2),(\varphi}(2)}\right) & =q(q+1) K_{2}\left(\varphi^{(2)}\right) .
\end{aligned}
$$

Let 1 and $S t$ be the trivial and the Steinberg representations of $U_{2}(q)$ respectively and let $\tilde{\varphi}=\varphi \circ$ det. Then $R_{\mathbf{T}_{(1,1),(\varphi, \varphi)}}=(-1+S t) \cdot \tilde{\varphi}$ and $R_{\mathbf{T}_{(2),(\varphi(2))}}=(-1-S t) \cdot \tilde{\varphi}$. Therefore we have

$$
\begin{aligned}
w(\tilde{\varphi}) & =q J(\varphi)^{2}-q^{2}(q+1) \varphi(-1), \\
w(S t \cdot \tilde{\varphi}) & =q J(\varphi)^{2}-q(q+1) \varphi(-1) .
\end{aligned}
$$

2.3. Symplectic groups. Let $\mathbf{G}=\left\{g \in G L_{2 n}\left(\overline{\mathbb{F}}_{q}\right): J={ }^{t} g J g\right\}$, where $J=$ $\left(\begin{array}{cc}0 & 1_{n} \\ -1_{n} & 0\end{array}\right)$ and $\sigma\left(\left(g_{i j}\right)\right)=\left(g_{i j}^{q}\right)$. Then $G=\mathbf{G}^{\sigma}$ is a symplectic group, denoted by $\operatorname{Sp}_{2 n}(q)$.

The $G$-conjugacy classes of $\sigma$-stable maximal tori of $\mathbf{G}$ are parametrized by the conjugacy classes of Weyl groups of type $C_{n}$ and hence correspond bijectively to the set of double partitions $\mathcal{P}_{n}^{(2)}$ of $n$, i.e. $\mathcal{P}_{n}^{(2)}=\{(\lambda, \mu): \lambda, \mu \in \mathcal{P},|\lambda|+|\mu|=n\}$.

Let $\mathbf{T}_{\lambda, \mu}$ be a maximal torus corresponding to a double partition $(\lambda, \mu) \in \mathcal{P}_{n}^{(2)}$, where $\lambda=\left(\lambda_{1}, \cdots, \lambda_{r}\right)$ and $\mu=\left(\mu_{1}, \cdots, \mu_{s}\right)$. Then $T_{\lambda, \mu}=\mathbf{T}_{\lambda, \mu}^{\sigma}$ is isomorphic to

$$
\prod_{i=1}^{r} \mathbb{F}_{q^{\lambda_{i}}}^{\times} \times \prod_{j=1}^{s} C_{\mu_{j}}
$$

Let $\pi_{i}$ and $\varphi_{j}$ be characters of $\mathbb{F}_{q^{\lambda_{i}}}^{\times}$and $C_{\mu_{j}}$, respectively, and $\theta$ be the character of $T_{\lambda, \mu}$ corresponding to $\left(\left(\pi_{i}\right),\left(\varphi_{j}\right)\right)$ under the isomorphism above.

Let $\rho$ be the canonical inclusion of $G$ into $G L_{2 n}(q)$ and $\psi(g)=\chi(\operatorname{tr} g)$ for $g \in G$. Then we have

PROPOSITION. With the notation as above

$$
\tau_{W}\left(R_{\mathbf{T}_{\lambda, \mu}, \theta}\right)=\left|G: T_{\lambda, \mu}\right| \prod_{i=1}^{r} K_{\lambda_{i}}\left(\pi_{i}\right) \times \prod_{j=1}^{s} J_{\mu_{j}}\left(\varphi_{j}\right) .
$$

In particular, if $\theta$ is in general position,

$$
w\left((-1)^{n+r} R_{\mathbf{T}_{\lambda, \mu}, \theta}\right)=(-1)^{n+r} q^{n^{2}} \prod_{i=1}^{r} K_{\lambda_{i}}\left(\pi_{i}\right) \times \prod_{j=1}^{s} J_{\mu_{j}}\left(\varphi_{j}\right) .
$$

EXAMPLE. $S p_{4}(q)$ Following is the table of $w\left(\chi_{R}\right)$ attached to $G=S p_{4}(q)$ and all irreducible characters $\chi_{R}$ of $G$, where we use the notation of Srinivasan in [16] for $\chi_{R}$. In the calculation we fully use the properties of unitary Kloosterman sums proved in [2]. 


\begin{tabular}{|c|c|c|}
\hline$\chi_{R}$ & $w\left(\chi_{R}\right)$ & \\
\hline$\chi_{1}\left(\varphi^{\prime}\right)$ & $q^{4} J_{2}\left(\varphi^{\prime}\right)$ & \\
\hline$\chi_{2}\left(\pi^{\prime}\right)$ & $q^{4} K_{2}\left(\pi^{\prime}\right)$ & \\
\hline$\chi_{3}\left(\pi_{1}, \pi_{2}\right)$ & $q^{4} K_{1}\left(\pi_{1}\right) K_{1}\left(\pi_{2}\right)$ & \\
\hline$\chi_{4}\left(\varphi_{1}, \varphi_{2}\right)$ & $q^{4} J_{1}\left(\varphi_{1}\right) J_{1}\left(\varphi_{2}\right)$ & \\
\hline$\chi_{5}(\pi, \varphi)$ & $q^{4} K_{1}(\pi) J_{1}(\varphi)$ & \\
\hline$\chi_{6}(\varphi)$ & $-q^{4} J_{1}(\varphi)^{2}$ & $+q^{5}(q+1) \varphi(-1)$ \\
\hline$\chi_{7}(\varphi)$ & $q^{4} J_{1}(\varphi)^{2}$ & $-q^{4}(q+1) \varphi(-1)$ \\
\hline$\chi_{8}(\pi)$ & $q^{4} K_{1}(\pi)^{2}$ & $+q^{5}(q-1) \pi(-1)$ \\
\hline$\chi_{9}(\pi)$ & $q^{4} K_{1}(\pi)^{2}$ & $-q^{4}(q-1) \pi(-1)$ \\
\hline$\xi_{1}(\varphi), \xi_{1}^{\prime}(\varphi)$ & $q^{4} K J_{1}(\varphi)$ & \\
\hline$\xi_{3}(\pi), \xi_{3}^{\prime}(\pi)$ & $q^{4} K K_{1}(\pi)$ & \\
\hline$\xi_{21}(\varphi), \xi_{22}(\varphi)$ & $q^{4} K_{1}\left(\pi_{0}\right) J_{1}(\varphi)$ & \\
\hline$\xi_{21}^{\prime}(\varphi), \xi_{22}^{\prime}(\varphi)$ & $q^{4} J_{1}(\varphi) J_{1}\left(\varphi_{0}\right)$ & \\
\hline$\xi_{41}(\pi), \xi_{42}(\pi)$ & $q^{4} K_{1}(\pi) K_{1}\left(\pi_{0}\right)$ & \\
\hline$\xi_{41}^{\prime}(\pi), \xi_{42}^{\prime}(\pi)$ & $q^{4} K_{1}(\pi) J_{1}\left(\varphi_{0}\right)$ & \\
\hline$\Phi_{1}, \Phi_{2}, \Phi_{3}, \Phi_{4}$ & $q^{4} K J_{1}\left(\varphi_{0}\right)$ & \\
\hline$\Phi_{5}, \Phi_{6}, \Phi_{7}, \Phi_{8}$ & $q^{4} K K_{1}\left(\pi_{0}\right)$ & \\
\hline$\Phi_{9}$ & $q^{4} K_{1}\left(\pi_{0}\right)^{2}$ & $+q^{4}(q-1)^{2} \pi_{0}(-1)$ \\
\hline$\theta_{1}, \theta_{2}$ & $q^{4} K_{1}\left(\pi_{0}\right)^{2}$ & $-2 q^{4}(q-1)^{2} \pi_{0}(-1)$ \\
\hline$\theta_{3}, \theta_{4}$ & $q^{4} K_{1}\left(\pi_{0}\right)^{2}$ & $+2 q^{5}(q-1) \pi_{0}(-1)$ \\
\hline$\theta_{5}, \theta_{6}, \theta_{7}, \theta_{8}$ & $q^{4} K_{1}\left(\pi_{0}\right) J_{1}\left(\varphi_{0}\right)$ & \\
\hline$\theta_{9}$ & $q^{4} K^{2}$ & $+q^{4}(q-1)^{2}$ \\
\hline$\theta_{10}$ & $q^{4} K^{2}$ & $-q^{4}(q+1)^{2}$ \\
\hline$\theta_{11}$ & $q^{4} K^{2}$ & $+q^{4}\left(q^{2}-1\right)$ \\
\hline$\theta_{12}$ & $q^{4} K^{2}$ & $-q^{4}\left(q^{2}-1\right)$ \\
\hline$\theta_{13}=S t$ & $q^{4} K^{2}$ & $-q^{3}\left(q^{2}-1\right)$ \\
\hline$\theta_{0}=1$ & $q^{4} K^{2}$ & $+q^{5}\left(q^{2}-1\right)$ \\
\hline
\end{tabular}

where $K=K_{1}(1), J=J_{1}(1)=-K$.

REMARKS. (i) If $G$ is a unitary group $U_{n}(q)$ or a symplectic group $S p_{2 n}(q)$, we can show that for a unipotent character $\chi_{R}$ we have

$$
w\left(\chi_{R}\right)=|G|_{p} K^{n}+\text { lower terms of } K \text { with coefficients in } \mathbb{Q}(q) .
$$

It is very plausible that these coefficients are in $Z[q]$ as can be seen in the example above. It is desirable to determine these coefficients explicitly.

(ii) We can argue similarly for special orthogonal groups, details of which are omitted. 


\section{Kloosterman sums attached to finite reductive groups.}

3.1. We use the same notation as in (2.1). For $a \in \mathbb{F}_{q}^{\times}$, let

$$
\psi_{a}(g)=\left(\chi \circ \operatorname{Tr}_{\mathbb{F}_{q^{m}} / \mathbb{F}_{q}}\right)\left(\operatorname{tr} \rho(g)+a \operatorname{tr} \rho\left(g^{-1}\right)\right) .
$$

In this case $W_{G}\left(R, \psi_{a}\right)$ is called the Kloosterman sum attached to $G, \rho$ and $R$. For unitary, symplectic and orthogonal groups with canonical inclusion $\rho$, Kloosterman sums become Gaussian sums, since $\operatorname{Tr}_{\mathbb{F}_{q^{2}} / \mathbb{F}_{q}}(\operatorname{tr} g)=\operatorname{Tr}_{\mathbb{F}_{q^{2}} / \mathbb{F}_{q}}\left(\operatorname{tr} g^{-1}\right)$ for unitary groups and $\operatorname{tr} g=\operatorname{tr} g^{-1}$ for symplectic and orthogonal groups.

3.2. Let $\mathbf{G}=G L_{n}\left(\overline{\mathbb{F}}_{q}\right), \sigma\left(\left(g_{i, j}\right)\right)=\left(g_{i, j}^{q}\right)$ for $\left(g_{i, j}\right) \in \mathbf{G}$, and hence $G=\mathbf{G}^{\boldsymbol{\sigma}}=$ $G L_{n}(q)$. The $G$-conjugacy classes of $\sigma$-stable maximal tori are parametrized by $\mathcal{P}_{n}$. Let $\mathbf{T}_{\lambda}$ be a maximal torus corresponding to $\lambda=\left(\lambda_{1}, \cdots, \lambda_{r}\right) \in \mathcal{P}_{n}$, then $T_{\lambda}=\mathbf{T}_{\lambda}^{\sigma}$ is isomorphic to $\prod_{i=1}^{r} \mathbb{F}_{q^{\lambda_{i}}}^{\times}$. Let $\theta$ be the character of $T$ corresponding to $\left(\pi_{i}\right)$ under this isomorphism above, where $\pi_{i}$ is a character of $\mathbb{F}_{q^{\lambda_{i}}}^{\times}$. Finally let $\rho$ be the identity map and so $\psi_{a}(g)=$ $\chi\left(\operatorname{tr} g+a \operatorname{tr} g^{-1}\right)$ in this case. Then by theorem (1.2), we have

PROPOSITION.

$$
\tau_{W}\left(R_{\mathbf{T}_{\lambda}, \theta}, \psi_{a}\right)=\left|G: T_{\lambda}\right| \prod_{i=1}^{r} K_{\lambda_{i}}\left(\pi_{i}, a\right)
$$

In particular, if $\theta$ is in general position,

$$
w\left((-1)^{n+r} R_{\mathbf{T}_{\lambda}, \theta}, \psi_{a}\right)=(-1)^{n+r} q^{\left(\begin{array}{c}
n \\
2
\end{array}\right)} \prod_{i=1}^{r} K_{\lambda_{i}}\left(\pi_{i}, a\right) .
$$

3.3. Now let us consider the values of Kloosterman sums for unipotent characters of $G=G L_{n}(q)$. For $\mu \in \mathcal{P}_{n}$, let $\chi^{\mu}$ be the corresponding irreducible character of $S_{n}$ defined in (7.4) of [14]. Also for $\lambda \in \mathcal{P}_{n}$, let $w_{\lambda}$ be a permutation in $S_{n}$ with cycle-type $\lambda$. We denote by $\chi_{\lambda}^{\mu}$ the value of $\chi^{\mu}$ at $w_{\lambda}$ and by $z_{\lambda}$ the order of the centralizer of $w_{\lambda}$ in $S_{n}$. Then every unipotent character $R_{\mu}$ of $G$ is obtained as follows:

$$
R_{\mu}=\sum_{\lambda \vdash n} z_{\lambda}^{-1} \chi_{\lambda}^{\mu} R_{\mathbf{T}_{\lambda}, 1}
$$

3.4. To calculate $w\left(R_{\mu}, \psi_{a}\right)$, we recall some properties of symmetric functions (cf. [14]). For each $\lambda \in \mathcal{P}_{n}$, let $p_{\lambda}(x)=p_{\lambda}\left(x_{1}, x_{2}, \cdots\right)$ be the power sum, $e_{\lambda}(x)=e_{\lambda}\left(x_{1}, x_{2}, \cdots\right)$ be the elementary symmetric functions and $s_{\lambda}(x)=s_{\lambda}\left(x_{1}, x_{2}, \cdots\right)$ be the Schur function. Each set $\left\{p_{\lambda}\right\}_{\lambda \vdash n},\left\{e_{\lambda}\right\}_{\lambda \vdash n}$ and $\left\{s_{\lambda}\right\}_{\lambda \vdash n}$ is a basis of the space of symmetric functions of degree $n$ and they are related as follows:

$$
p_{\lambda}(x)=\sum_{\nu \vdash n} \chi_{\lambda}^{\nu} s_{\nu}(x), \quad s_{\nu}(x)=\sum_{\kappa \vdash n} a_{\nu \kappa} e_{\kappa}(x),
$$

where $\left(a_{\nu \kappa}\right)=J K^{*}$ (for the definition of matrices $J$ and $K^{*}$, see (I.6) of [14]).

From Theorem 1 of [2], we have

$$
K_{m}(1, a)=-\alpha^{m}-\beta^{m},
$$


where $\alpha, \beta$ are complex numbers determined by the relations $K_{1}(1, a)=-\alpha-\beta$ and $q=\alpha \beta$. Thus $K_{\lambda_{i}}(1, a)=-p_{\lambda_{i}}(\alpha, \beta, 0, \cdots)$.

For the symmetric function $f(x)=f\left(x_{1}, x_{2}, \cdots\right)$, we shall write $f=f(\alpha, \beta, 0, \cdots)$. For example $e_{1}=\alpha+\beta, e_{2}=\alpha \beta$ and $e_{k}=0$ if $k \geq 3$. With this convention, by (3.2) we have

$$
\tau_{W}\left(R_{\mathbf{T}_{\lambda}, 1}, \psi_{a}\right)=(-1)^{\ell(\lambda)}\left|G: T_{\lambda}\right| p_{\lambda}=(-1)^{n}|G|_{p} R_{\mathbf{T}_{\lambda}, 1}(1) p_{\lambda}
$$

where the second equation follows since $R_{\mathbf{T}_{\lambda}, 1}(1)=(-1)^{n-\ell(\lambda)}\left|G: T_{\lambda}\right|_{p^{\prime}}$ and $\left|G: T_{\lambda}\right|_{p}=$ $|G|_{p}$.

Moreover in $[15,7.2]$ it is shown that

$$
R_{\mathbf{T}_{\lambda}, 1}(1)=\sum_{i=0}^{\left(\begin{array}{l}
n \\
2
\end{array}\right)} \rho_{\lambda}^{i} q^{i}
$$

where $\rho^{i}$ is the character of the representation of $S_{n}$ given by the homogeneous subspace of degree $i$ of the coinvariant algebra of $S_{n}$ and $\rho_{\lambda}^{i}$ is its value at the element with cycle type $\lambda$. Combining these informations together, we have the following

Proposition.

$$
\left.w\left(R_{\mu}, \psi_{a}\right)=\frac{(-1)^{n} q^{\left(\begin{array}{c}
n \\
2
\end{array}\right)}}{\operatorname{deg} R_{\mu}} \sum_{\substack{\kappa \vdash n \\
l\left(\kappa^{\prime}\right) \leq 2}} \sum_{\substack{\nu \vdash n \\
0 \leq i \leq\left(\begin{array}{c}
n \\
2
\end{array}\right)}}\left\langle\chi^{\mu} \chi^{\nu}, \rho^{i}\right\rangle a_{\nu \kappa} q^{i}\right) e_{\kappa} .
$$

3.5. Let $(-1)^{n} f_{\mu}^{\kappa}(q)$ be the coefficient of $e_{\kappa}$ in the right hand side of (3.4.1). Hence

$$
f_{\mu}^{\kappa}(q)=\frac{q^{\left(\begin{array}{c}
n \\
2
\end{array}\right)}}{\operatorname{deg} R_{\mu}} \sum_{\substack{\nu \vdash n \\
0 \leq i \leq\left(\begin{array}{c}
n \\
2
\end{array}\right)}}\left\langle\chi^{\mu} \chi^{\nu} ; \rho^{i}\right\rangle a_{\nu \kappa} q^{i}
$$

and

$$
w\left(R_{\mu}, \psi_{a}\right)=(-1)^{n} \sum_{\substack{\kappa \vdash n \\ l\left(\kappa^{\prime}\right) \leq 2}} f_{\mu}^{\kappa}(q) e_{\kappa} .
$$

3.6. PROPOSITION. For $\kappa, \mu \vdash n$, the following properties of $f_{\mu}^{\kappa}(q)$ hold.

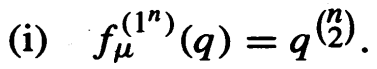

(ii) $f_{\mu}^{\kappa}(1)=0$, if $\kappa \neq\left(1^{n}\right)$.

(iii) $q^{\left(\begin{array}{c}n \\ 2\end{array}\right)} f_{\mu}^{\kappa}\left(q^{-1}\right)=f_{\mu^{\prime}}^{\kappa}(q)$, where $\mu^{\prime}$ is the dual partition of $\mu$.

PROOF. (i) By (3.5.1) and the fact that

$$
a_{v,\left(1^{n}\right)}= \begin{cases}1, & \text { if } v=(n) \\ 0, & \text { if } v \neq(n)\end{cases}
$$


we have

$$
f_{\mu}^{\left(1^{n}\right)}(q)=\frac{q^{\left(\begin{array}{c}
n \\
2
\end{array}\right)}}{\operatorname{deg} R_{\mu}} \sum_{0 \leq i \leq\left(\begin{array}{l}
n \\
2
\end{array}\right)}\left\langle\chi^{\mu}, \rho^{i}\right\rangle q^{i}
$$

Then the required result follows, since

$$
\operatorname{deg} R_{\mu}=\sum_{0 \leq i \leq\left(\begin{array}{c}
n \\
2
\end{array}\right)}\left\langle\chi^{\mu}, \rho^{i}\right\rangle q^{i} .
$$

(ii) By putting $q=1$ in (3.5.1), we have

$$
f_{\mu}^{\kappa}(1)=\frac{1}{\chi_{\left(1^{n}\right)}^{\mu}} \sum_{\substack{\nu \vdash n \\
0 \leq i \leq\left(\begin{array}{c}
n \\
2
\end{array}\right)}}\left\langle\chi^{\mu} \chi^{\nu}, \rho^{i}\right\rangle a_{\nu \kappa}
$$

Define the nonnegative integers $\gamma_{\mu \nu}^{\lambda}$, by

$$
\chi^{\mu} \chi^{\nu}=\sum_{\lambda \vdash n} \gamma_{\mu \nu}^{\lambda} \chi^{\lambda}
$$

Then

$$
\sum_{0 \leq i \leq\left(\begin{array}{l}
n \\
2
\end{array}\right)}\left\langle\chi^{\mu} \chi^{\nu}, \rho^{i}\right\rangle=\sum_{\substack{\lambda \vdash n \\
0 \leq i \leq\left(\begin{array}{c}
n \\
2
\end{array}\right)}} \gamma_{\mu \nu}^{\lambda}\left\langle\chi^{\lambda}, \rho^{i}\right\rangle=\sum_{\lambda \vdash n} \gamma_{\mu \nu}^{\lambda} \chi_{\left(1^{n}\right)}^{\lambda}=\chi_{\left(1^{n}\right)}^{\mu} \chi_{\left(1^{n}\right)}^{\nu} .
$$

So we have

$$
f_{\mu}^{\nu}(1)=\sum_{\nu \vdash n} \chi_{\left(1^{n}\right)}^{\nu} a_{\nu \kappa}
$$

On the other hand

$$
e_{\left(1^{n}\right)}(x)=p_{\left(1^{n}\right)}(x)=\sum_{\kappa, \nu \vdash n} \chi_{\left(1^{n}\right)}^{\nu} a_{\nu \kappa} e_{\kappa}(x)=\sum_{\kappa \vdash n} f_{\mu}^{\kappa}(1) e_{\kappa}(x) .
$$

By comparing the coefficient of $e_{\kappa}(x), \kappa \neq\left(1^{n}\right)$, on both hands, we have the required results.

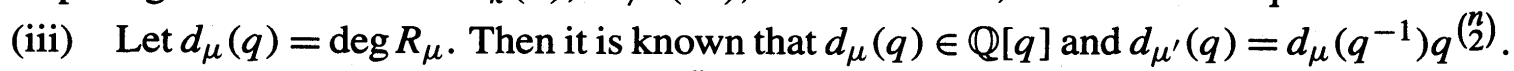
Moreovedr $\chi^{\mu^{\prime}}=\chi^{\left(1^{n}\right)} \chi^{\mu}$ and $\chi^{\left(1^{n}\right)} \rho^{i}=\rho^{\left(\begin{array}{c}n \\ 2\end{array}\right)-i}$. Using these facts and (3.5.1) we can show (iii).

3.7. We have computed $f_{\mu}^{\kappa}(q)$ for all $\kappa, \mu \vdash n$, if $n \leq 7$. From these computations we conjecture that $f_{\mu}^{\kappa}(q) \in \mathbb{Z}[q]$. For $\kappa=\left(2,1^{n-2}\right)$, we can give more precise conjecture as follows; let $\mu$ be given as $\left(\alpha_{1}, \cdots, \alpha_{r} \mid \beta_{1}, \cdots, \beta_{r}\right)$ by the Frobenius notation (cf. p. 3 of [14]), and $\alpha^{\prime}$ and $\beta^{\prime}$ be the conjugates of $\alpha$ and $\beta$, respectively. Then the following equation will hold:

$$
f_{\mu}^{\left(2,1^{n-2}\right)}(q)=\sum_{i=1}^{\beta_{1}} \beta_{i}^{\prime} q^{\left(\begin{array}{c}
n \\
2
\end{array}\right)-i}-(n-r) q^{\left(\begin{array}{c}
n \\
2
\end{array}\right)}+\sum_{i=1}^{\alpha_{1}} \alpha_{i}^{\prime} q^{\left(\begin{array}{c}
n \\
2
\end{array}\right)+i} .
$$

3.8. Here we give the table of $(-1)^{n} f_{\mu}^{\kappa}(q)$ for $n \leq 6$. The numbers $d$ in the second row of each table are exponents in $q$ and each entry represents the coefficient of $q^{d}$ in $(-1)^{n} f_{\mu}^{\kappa}(q)$. 
Empty entry represents 0 . Thus for example for $n=4$ and $\mu=(3,1)$, we can read from the table that

$$
w\left(R_{(3,1)}, \psi_{a}\right)=q^{6} e_{\left(1^{4}\right)}+\left(q^{5}-3 q^{6}+q^{7}+q^{8}\right) e_{\left(2,1^{2}\right)}+\left(-q^{5}+2 q^{6}-q^{7}\right) e_{\left(2^{2}\right)} .
$$

$n=2$

\begin{tabular}{c|c|ccc}
\multirow{2}{*}{$\mu$} & $e_{\left(1^{2}\right)}$ & \multicolumn{3}{|c}{$e_{(2)}$} \\
\cline { 2 - 5 }$\mu$ & 1 & 0 & 1 & 2 \\
\hline 2 & 1 & & -1 & 1 \\
\hline $1^{2}$ & 1 & 1 & -1 & \\
\hline
\end{tabular}

$$
n=3
$$

\begin{tabular}{|c|c|c|c|c|c|c|}
\hline \multirow[b]{2}{*}{$\mu$} & \multirow{2}{*}{$\frac{e_{\left(1^{3}\right)}}{3}$} & \multicolumn{5}{|c|}{$e_{(2,1)}$} \\
\hline & & 1 & 2 & 3 & 4 & 5 \\
\hline 3 & -1 & & & 2 & -1 & -1 \\
\hline 2,1 & -1 & & -1 & 2 & -1 & \\
\hline $1^{3}$ & -1 & -1 & -1 & 2 & & \\
\hline
\end{tabular}

\begin{tabular}{|c|c|c|c|c|c|c|c|c|c|c|c|c|c|c|c|c|c|}
\hline \multirow[b]{2}{*}{$\mu$} & \multirow{2}{*}{$\frac{e_{\left(1^{4}\right)}}{6}$} & \multicolumn{7}{|c|}{$e_{\left(2,1^{2}\right)}$} & \multicolumn{9}{|c|}{$e_{\left(2^{2}\right)}$} \\
\hline & & 3 & 4 & 5 & 6 & 7 & 8 & 9 & 2 & 3 & 4 & 5 & 6 & 7 & 8 & 9 & 10 \\
\hline 4 & 1 & & & & -3 & 1 & 1 & 1 & & & & & 1 & -1 & & -1 & 1 \\
\hline 3,1 & 1 & & & 1 & -3 & 1 & 1 & & & & & -1 & 2 & -1 & & & \\
\hline $2^{2}$ & 1 & & & 1 & -2 & 1 & & & & & 1 & -1 & & -1 & 1 & & \\
\hline $2,1^{2}$ & 1 & & 1 & 1 & -3 & 1 & & & & & & -1 & 2 & -1 & & & \\
\hline $1^{4}$ & 1 & 1 & 1 & 1 & -3 & & & & 1 & -1 & & -1 & 1 & & & & \\
\hline
\end{tabular}

$n=4$

$$
n=5
$$

\begin{tabular}{c|c|ccccccccc} 
& $e_{\left(1^{5}\right)}$ & \multicolumn{10}{|c}{$e_{\left(2,1^{3}\right)}$} \\
\cline { 2 - 10 }$\mu$ & 10 & 6 & 7 & 8 & 9 & 10 & 11 & 12 & 13 & 14 \\
\hline 5 & -1 & 1 & & & 4 & -1 & -1 & -1 & -1 \\
\hline 4,1 & -1 & & & -1 & 4 & -1 & -1 & -1 & \\
\hline 3,2 & -1 & & & -1 & 3 & -1 & -1 & & \\
\hline $3,1^{2}$ & -1 & & -1 & -1 & 4 & -1 & -1 & & \\
\hline $2^{2}, 1$ & -1 & & -1 & -1 & 3 & -1 & & & \\
\hline $2,1^{3}$ & -1 & & -1 & -1 & -1 & 4 & -1 & & & \\
\hline $1^{5}$ & -1 & -1 & -1 & -1 & -1 & 4 & & & & \\
\hline
\end{tabular}




\begin{tabular}{|c|c|c|c|c|c|c|c|c|c|c|c|c|c|}
\hline \multirow[b]{2}{*}{$\mu$} & \multicolumn{13}{|c|}{$e_{\left(2^{2}, 1\right)}$} \\
\hline & 4 & 5 & 6 & 7 & 8 & 9 & 10 & 11 & 12 & 13 & 14 & 15 & 16 \\
\hline 5 & & & & & & & -3 & 2 & 1 & 1 & 1 & -1 & -1 \\
\hline 4,1 & & & & & & 2 & -4 & 1 & 1 & 1 & -1 & & \\
\hline 3,2 & & & & & -1 & 2 & -2 & 1 & 1 & -1 & & & \\
\hline $3,1^{2}$ & & & & & 1 & 1 & -4 & 1 & 1 & & & & \\
\hline $2^{2}, 1$ & & & & -1 & 1 & 1 & -2 & 2 & -1 & & & & \\
\hline $2,1^{3}$ & & & -1 & 1 & 1 & 1 & -4 & 2 & & & & & \\
\hline $1^{5}$ & -1 & -1 & 1 & 1 & 1 & 2 & -3 & & & & & & \\
\hline
\end{tabular}

$n=6$

\begin{tabular}{|c|c|c|c|c|c|c|c|c|c|c|c|c|}
\hline \multirow[b]{2}{*}{$\mu$} & \multirow{2}{*}{$\frac{e_{\left(1^{6}\right)}}{15}$} & \multicolumn{11}{|c|}{$e_{\left(2,1^{4}\right)}$} \\
\hline & & 10 & 11 & 12 & 13 & 14 & 15 & 16 & 17 & 18 & 19 & 20 \\
\hline 6 & 1 & & & & & & -5 & 1 & 1 & 1 & 1 & 1 \\
\hline 5,1 & 1 & & & & & 1 & -5 & 1 & 1 & 1 & 1 & \\
\hline 4,2 & 1 & & & & & 1 & -4 & 1 & 1 & 1 & & \\
\hline $4,1^{2}$ & 1 & & & & 1 & 1 & -5 & 1 & 1 & 1 & & \\
\hline $3^{2}$ & 1 & & & & & 1 & -4 & 2 & 1 & & & \\
\hline $3,2,1$ & 1 & & & & 1 & 1 & -4 & 1 & 1 & & & \\
\hline $3,1^{3}$ & 1 & & & 1 & 1 & 1 & -5 & 1 & 1 & & & \\
\hline $2^{3}$ & 1 & & & & 1 & 2 & -4 & 1 & & & & \\
\hline $2^{2}, 1^{2}$ & 1 & & & 1 & 1 & 1 & -4 & 1 & & & & \\
\hline $2,1^{4}$ & 1 & & 1 & 1 & 1 & 1 & -5 & 1 & & & & \\
\hline $1^{6}$ & 1 & 1 & 1 & 1 & 1 & 1 & -5 & & & & & \\
\hline
\end{tabular}




\begin{tabular}{|c|c|c|c|c|c|c|c|c|c|c|c|c|c|c|c|c|c|}
\hline \multirow[b]{2}{*}{$\mu$} & \multicolumn{17}{|c|}{$e_{\left(2^{2}, 1^{2}\right)}$} \\
\hline & 7 & 8 & 9 & 10 & 11 & 12 & 13 & 14 & 15 & 16 & 17 & 18 & 19 & 20 & 21 & 22 & 23 \\
\hline 6 & & & & & & & & & 6 & -3 & -2 & -2 & -1 & -2 & 2 & 1 & 1 \\
\hline 5,1 & & & & & & & & -3 & 7 & -2 & -1 & -2 & -1 & 1 & 1 & & \\
\hline 4,2 & & & & & & & 1 & -3 & 4 & -1 & -1 & -2 & 2 & & & & \\
\hline $4,1^{2}$ & & & & & & & -2 & -2 & 8 & -2 & -1 & -2 & 1 & & & & \\
\hline $3^{2}$ & & & & & & & 1 & -2 & 5 & -5 & -1 & 1 & 1 & & & & \\
\hline $3,2,1$ & & & & & & 1 & -2 & -1 & 4 & -1 & -2 & 1 & & & & & \\
\hline $3,1^{3}$ & & & & & 1 & -2 & -1 & -2 & 8 & -2 & -2 & & & & & & \\
\hline $2^{3}$ & & & & & 1 & 1 & -1 & -5 & 5 & -2 & 1 & & & & & & \\
\hline $2^{2}, 1^{2}$ & & & & & 2 & -2 & -1 & -1 & 4 & -3 & 1 & & & & & & \\
\hline $2,1^{4}$ & & & 1 & 1 & -1 & -2 & -1 & -2 & 7 & -3 & & & & & & & \\
\hline $1^{6}$ & 1 & 1 & 2 & -2 & -1 & -2 & -2 & -3 & 6 & & & & & & & & \\
\hline
\end{tabular}

\begin{tabular}{|c|c|c|c|c|c|c|c|c|c|c|c|c|c|c|c|c|c|c|c|}
\hline \multirow[b]{2}{*}{$\mu$} & \multicolumn{19}{|c|}{$e_{\left(2^{3}\right)}$} \\
\hline & 6 & 7 & 8 & 9 & 10 & 11 & 12 & 13 & 14 & 15 & 16 & 17 & 18 & 19 & 20 & 21 & 22 & 23 & 24 \\
\hline 6 & & & & & & & & & & -1 & 1 & & 1 & -1 & 1 & -1 & & -1 & 1 \\
\hline 5,1 & & & & & & & & & 1 & -2 & 1 & -1 & 2 & -1 & & & & & \\
\hline 4,2 & & & & & & & & -1 & 2 & -1 & & & 1 & -2 & 1 & & & & \\
\hline $4,1^{2}$ & & & & & & & & & 1 & -2 & 1 & -1 & 2 & -1 & & & & & \\
\hline $3^{2}$ & & & & & & & 1 & -1 & & -2 & 2 & & 1 & -1 & & & & & \\
\hline $3,2,1$ & & & & & & & & & & & & & & & & & & & \\
\hline $3,1^{3}$ & & & & & & -1 & 2 & -1 & 1 & -2 & 1 & & & & & & & & \\
\hline $2^{3}$ & & & & & & -1 & 1 & & 2 & -2 & & -1 & 1 & & & & & & . \\
\hline $2^{2}, 1^{2}$ & & & & & 1 & -2 & 1 & & & -1 & 2 & -1 & & & & & & & \\
\hline $2,1^{4}$ & & & & & & -1 & 2 & -1 & 1 & -2 & 1 & & & & & & & & \\
\hline $1^{6}$ & 1 & -1 & & -1 & 1 & -1 & 1 & & 1 & -1 & & & & & & & & & \\
\hline
\end{tabular}

\section{References}

[ 1 ] R. W. CARTER, Finite groups of Lie type, Interscience (1985).

[ 2 ] C. W. CURTIS and K. SHINODA, Unitary Kloosterman sums and Gelfand-Graev representation of $G L_{2}, J$. Algebra 216 (1999), 431-447.

[ 3 ] P. Deligne and G. Lusztig, Representations of reductive groups over finite fields, Ann. of Math. 103 (1976), 103-161. 
[ 4 ] D. S. KIM and I. LEE, Gauss sums for $O^{+}(2 n, q)$, Acta Arith. 78 (1996), 75-89.

[ 5 ] D. S. KIM, Gauss sums for $O^{-}(2 n, q)$, Acta Arith. 80 (1997), 343-365.

[6] D. S. KIM, Gauss sums for general and special linear groups over a finite field, Arch. Math. 69 (1997), 297-304.

[ 7 ] D. S. KIM, Gauss sums for orthogonal groups over a finite field of characteristic two, Acta Arith. 82 (1997), 331-357.

[ 8 ] D. S. KIM, Gauss sums for $U\left(2 n+1, q^{2}\right)$, J. Korean Math. Soc. 34 (1997), 871-894.

[ 9 ] D. S. KIM, Gauss sums for $O(2 n+1, q)$, Finite Fields Appl. 4 (1998), 62-86.

[10] D. S. KIM, Gauss sums for $U\left(2 n, q^{2}\right)$, Glasgow Math. J. 40 (1998), 79-95.

[11] D. S. KIM, Gauss sums for symplectic groups over a finite field, Monatsh. Math. 126 (1998), 55-71.

[12] T. KoNDO, On Gaussian sums attached to the general linear groups over finite fields, J. Math. Soc. Japan 15 (1963), 244-255.

[13] I. LEE and K. PARK, Gauss sums for $G_{2}(q)$, Bull. Korean Math. Soc. 34 (1997), 305-315.

[14] I. G. MACDONALD, Symmmetric functions and Hall polynomials, Second ed., Clarendon Press (1995).

[15] T. A. SPRINGER, Trigonometric sums, Green functions of finite groups and representations of Weyl groups, Invent. Math. 36 (1976), 173-207.

[16] B. SRINIVASAN, The characters of the finite symplectic group $S p(4, q)$, Trans. Amer. Math. Soc. 131 (1968), 488-525.

Present Address:

DEPARTMENT OF MATHEMATICS, SOPHIA UNIVERSITY, KIOICHO, CHIYODA-KU, TOKYO 102-8554, JAPAN.

e-mail: saitou-n@mm.sophia.ac.jp shinoda@mm.sophia.ac.jp 